\title{
Nuclear Medicine Training in the United States
}

\author{
George M. Segall, Erin E. Grady, Joanna R. Fair, Munir V. Ghesani, and Leonie Gordon \\ American Board of Nuclear Medicine, St. Louis, Missouri
}

$\mathbf{I}_{n}$ the October issue of The Journal of Nuclear Medicine, the authors of the "Hot Topics" paper, "Nuclear Medicine Training: What Now?" suggested a path forward for training in nuclear medicine that "matches the needs of the evolving clinical specialty" by "combined, multispecialty training." (1). The American Board of Nuclear Medicine (ABNM) also endorses combined, multispecialty training that maintains high standards for nuclear medicine education, prepares physicians for practice in a changing environment, and advances the specialty.

The ABNM has actively supported training leading to certification in nuclear medicine plus certification by the American Board of Radiology (ABR) in diagnostic radiology. The ABNM and the Society of Nuclear Medicine and Molecular Imaging (SNMMI) issued a joint policy statement in 2012 supporting all training pathways leading to dual certification (2). These pathways currently include the traditional 12-mo nuclear radiology or nuclear medicine fellowship after diagnostic radiology residency, the 16-mo nuclear medicine/nuclear radiology pathway during $4 \mathrm{y}$ of diagnostic radiology residency, and the 5-y combined pathway within separate nuclear medicine and diagnostic radiology programs accredited by the Accreditation Council for Graduate Medical Education (ACGME). Support for these pathways is not meant to promote the "chaos of multiple training and certification pathways" but rather to facilitate institutions developing practical dual training pathways given local considerations, with robust training and subsequent certification in nuclear medicine and diagnostic radiology.

After the joint ABNM and SNMMI statement in 2012, the ABNM took action to promote the development of dual training programs within $4 \mathrm{y}$ of diagnostic radiology residency training. The changes made by the ABNM include the following:

- Defining 16 mo of training in practical terms.

- Removing the requirement for a minimum of 6 mo of continuous nuclear medicine training.

- Giving training-program directors more discretion in allowing elective time.

- Permitting contemporaneous nuclear medicine training with other fellowship training.

- Recognizing non-ACGME-accredited nuclear medicine fellowship training in institutions with ACGME-accredited nuclear medicine residency or nuclear radiology fellowship programs.

Received Sep. 11, 2017; revision accepted Sep. 13, 2017.

For correspondence or reprints contact: George M. Segall, Nuclear

Medicine Service 115, 3801 Miranda Ave., Palo Alto, CA 94304

E-mail: george.segall@va.gov

Published online Oct. 6, 2017

COPYRIGHT @ 2017 by the Society of Nuclear Medicine and Molecular Imaging.

DOI: 10.2967/jnumed.117.200857

- Recognizing nuclear radiology training when ACGME nuclear medicine program requirements are met.

The ABNM recognizes that it is a challenge for physicians to become proficient in nuclear medicine and diagnostic radiology in 16 and $32 \mathrm{mo}$, respectively. Recognizing also that the knowledge and skills required to advance the specialty of nuclear medicine and molecular imaging might require more than $4 \mathrm{y}$ of training, the ABNM also supports 5-y combined training programs with nuclear medicine and diagnostic radiology residency programs that are separately accredited by the ACGME. The ABNM gives institutions broad leeway in determining how much nuclear medicine and diagnostic radiology training is required, so long as the minimum time of nuclear medicine training is $16 \mathrm{mo}$. The ABNM has already approved combined training programs at several institutions, including 3 institutions that have a number issued by the National Residency Matching Program that allows applicants to simultaneously apply for the separately accredited nuclear medicine and diagnostic radiology residency programs (Stanford University, University of California at Davis, and Johns Hopkins University). The ACGME will also issue an Accreditation Data System program number, which acknowledges the board-approved combined program with separately accredited components. More details are available in Tracers, the newsletter sent to all ABNM diplomates (3).

The ABNM also makes it possible for radiologists trained outside the United States and Canada to qualify for the ABNM certification examination through the ABR alternate pathway, when it includes at least 16 mo of nuclear medicine training at institutions with ACGME-accredited nuclear medicine or nuclear radiology programs.

In June 2017, the ABNM sent a letter to all nuclear medicine and nuclear radiology program directors, describing in detail the dual training pathways diagnostic radiologists may follow to become ABNM-certified. The letter is available on the ABNM website (4).

Recognizing that maintenance of certification is more difficult for physicians certified by more than one American Board of Medical Specialties member board, the ABNM has taken steps to reduce the required time and effort by giving credit for the continuing medical education (maintenance of certification, part 2) and improvement-in-medical-practice activities (maintenance of certification, part 4) recognized by other member boards. The ABNM has also adopted diplomate self-attestation with random auditing in place of routinely requiring documentation of these activities (5).

The authors state that the nuclear medicine and diagnostic radiology communities are the imaging-world equivalent of the Dr. Seuss characters called the Zax, who, "marching straight 
ahead, came face to face" and "refused to budge, stopping the forward progress for both of them." This characterization discounts changes both boards have made to promote dual training, continuing to build on a foundation that includes $47 \%$ of ABNM diplomates certified between 2001 and 2016 who are also certified by the ABR.

The challenge for the specialty of nuclear medicine is to attract highly qualified medical students into the field. The number of nuclear medicine residency training programs has decreased from 56 in 2007-2008 to 42 in 2017-2018, with 80 residents currently in training. The number of nuclear radiology programs has decreased from 22 to 18 during the same period, with 12 trainees this year (6). In 2016, 42 physicians were newly certified by the ABNM, and 2 physicians were certified by the ABR in nuclear radiology. The SNMMI and the American College of Radiology have recognized the problem and have started efforts to reach out and educate young professionals about opportunities in the rapidly growing fields of nuclear medicine and nuclear radiology.

Nuclear medicine and molecular imaging have seen tremendous growth and have become more complex with the introduction of many new diagnostic and therapeutic techniques in clinical practice. The ABNM believes, therefore, that a training pathway with only 4 mo of nuclear medicine will not advance the specialty. On the other hand, 3 y of nuclear medicine residency training leading only to ABNM certification may not provide diplomates with adequate employment opportunities. The ABNM, therefore, supports pathways leading to certification in nuclear medicine and diagnostic radiology that provide multiple options for entry, as well as matching training with future professional goals.

The ABNM also recognizes the historical contributions to nuclear medicine made by physicians in other specialties and plans to maintain practical training pathways that allow these physicians to continue to enrich and expand the specialty. In the past $16 \mathrm{y}, 12 \%(133 / 1,116)$ of ABNM diplomates have been certified by non-ABR or American Board of Medical Specialties member boards, but that percentage has fallen to $4 \%$ in recent years. Time will tell how important these pathways will continue to be.

The authors state the "need for cross-fertilization in highly specialized areas of the practice such as nuclear cardiology and radionuclide therapy/theranostics." They envision a training pathway for combined radiation oncology and nuclear medicine. Others also recognize the need for combined multispecialty training. In the opening plenary session of the 2017 SNMMI annual meeting, Johannes Czernin, professor of molecular and medical pharmacology at the University of California, Los Angeles, and editor-in-chief of The Journal of Nuclear Medicine, gave a presentation titled, "Imaging with a Purpose: The Future of Nuclear Medicine, Molecular Imaging, and Therapy." He stated it was necessary to "recruit the best medical students and residents" and called on the ABNM to recreate a feasible and reasonable pathway for internal medicine trainees (joint program). He stated that nuclear medicine should solidify its independence by keeping itself equidistant from medicine, endocrinology, oncology, neurology, urology, radiology, and radiation oncology. The ABNM is reevaluating the combined residency training program in internal medicine and nuclear medicine that has been inactive for over a decade. It will also explore other combined multispecialty training pathways that may be needed for the future.

The authors rhetorically ask whether nuclear medicine physicians will get stuck in their own tracks when facing their radiology (and cardiology and radiation oncology) colleagues, refusing to budge while the rest of medicine builds roads around, over, and through them. The actions of the ABNM show that cooperation rather than confrontation is ABNM policy. As a nuclear medicine leader, the ABNM will continue to work with all stakeholders, including the $\mathrm{ABR}$ and other American Board of Medical Specialties member boards, the SNMMI, and the ACGME, to ensure that future physicians will have the education and training needed for nuclear medicine to thrive for the benefit of patients and physicians.

\section{REFERENCES}

1. Mankoff D, Pryma DA. Nuclear medicine training: what now? J Nucl Med. 2017;58:1536-1538.

2. Delbeke D, Royal HD, Frey KA, Graham MM, Segall GM. SNMMI/ABNM joint position statement on optimizing training in nuclear medicine in the era of hybrid imaging. J Nucl Med. 2012;53:1490-1494.

3. Iagaru A. Establishing a dual training pathway in nuclear medicine and diagnostic radiology: the Stanford experience. ABNM website. https://abnm_wordpress_ uploads.s3.amazonaws.com/wordpress/wp-content/uploads/2014-1_Establishing_ Dual_Pathway.pdf. Accessed September 27, 2017.

4. DR certified requirements. ABNM website. https://www.abnm.org/index.php/drcertified-requirements/. Accessed October 11, 2017.

5. Segall G. From the ABNM: making MOC simpler. J Nucl Med. 2017;58(6):17N.

6. Number of Accredited Programs, Academic Year 2017-2018, United States. ACGME website. https://apps.acgme.org/ads/Public/Reports/ReportRun? ReportId $=3 \&$ CurrentYear $=2017 \&$ Academic YearId $=2017$. Accessed September 27, 2017. 